de Mecánica Computacional

Mecánica Computacional Vol XXXII, págs. 3121-3132 (artículo completo)

Carlos G. García Garino, Aníbal E. Mirasso, Mario A. Storti, Miguel E. Tornello (Eds.)

Mendoza, Argentina, 19-22 Noviembre 2013

\title{
SYMMETRY BREAKING INSTABILITY IN A MIXED CONVECTION PROBLEM
}

\author{
J.C. Cajas ${ }^{\mathrm{a}}$, L. Martínez-Suástegui ${ }^{\mathrm{b}}$ and C. Treviño ${ }^{\mathrm{c}}$ \\ ${ }^{a}$ Facultad de Ciencias, Universidad Nacional Autónoma de México, México D.F., 04510, México, \\ jc.cajas@gmail.com \\ ${ }^{\mathrm{b}}$ ESIME Azcapotzalco, Instituto Politécnico Nacional, Avenida de las Granjas No. 682, Colonia Santa \\ Catarina, Delegación Azcapotzalco, México, Distrito Federal 02250, martinezlorenzo@gmail.com \\ ${ }^{\mathrm{c}}$ Facultad de Ciencias, UMDI Universidad Nacional Autónoma de México Sisal, Yucatán, Mexico, \\ ctrev@correo.unam.mx
}

Keywords: Stability Analysis, Oscillating Flow, Mixed convection

\begin{abstract}
In this work, the stability of a strongly non-parallel symmetrical counterflow mixed convection problem is studied, using numerically generated eigenfunctions. The base flow is numerically obtained for each value of the buoyancy parameter (Richardson number), and the stability of this flow is analyzed by increasing its value while all the others remained fixed. The perturbed linear functions are numerically generated by introducing a transient modulated asymmetrical buoyancy, relaxing at later times to 'numerical eigenfunctions'. The time evolution of the amplitude of these perturbations is used to obtain the stability characteristics. Symmetry breaking instability occurs, for fixed geometry, Reynolds and Prandtl numbers, for values of the buoyancy parameter larger than a critical one. However, there is also a window for the buoyancy parameter below this critical value, where the system shows instability, producing a slightly asymmetric thermal and flow response.
\end{abstract}




\section{INTRODUCTION}

Mixed convection is defined as heat transfer situations where both natural and forced convection mechanisms interact. In particular, the oscillatory behavior in mixed convection flows is of great interest because of its rich dynamical features and useful results to applied problems. Chang and Lin (1993) studied the steady laminar and transient oscillatory mixed convection in a symmetrically heated vertical plane channel, subjected to an opposing buoyancy assuming a fully developed velocity profile at the inlet and discrete heat sources that are maintained at uniform and equal heat fluxes. The authors pointed out that an oscillatory flow with a single fundamental frequency is found when the buoyancy parameter, or Richardson number, exceeds a critical value. Lin et al. (1993) investigated numerically the detailed flow and thermal characteristics in transient laminar opposing mixed convection in a vertical plane channel subjected to a symmetrical heat input. Their results show that at high opposing buoyancy, sudden flow asymmetry and oscillation occur simultaneously in an early steady flow after the initial transient. Evans and Greif (1997) showed the strong effects of buoyancy, even for small temperature differences, on the downward flow of nitrogen in a partially heated tall vertical channel and reported time-dependent oscillations, including periodic flow reversals along the channel walls. Martínez-Suástegui and Treviño (2007); Martínez-Suástegui et al. (2011) investigated the transient laminar mixed convection in an asymmetrically and differentially heated vertical channel of finite length subjected to an opposing buoyancy. Their results show that a final steady or oscillatory flow response is obtained depending on the value of the Reynolds and Richardson numbers, and that the critical value of the buoyancy strength between the two regimes strongly depends on the value of the Reynolds number.

Stability analyses in natural and mixed convection flows have been developed through the years, since they provide further insight of the instability mechanisms present in such situations and give quantitative information about the defining and critical parameters involved. Ever since the pioneering work of Lorenz (1963), who studied the instability of finite systems of deterministic ordinary nonlinear differential equations representing forced dissipative hydrodynamic flows, stability analyses of fluid flow phenomena experienced a rapid growth and became the starting point of the chaos theory. Carey and Gebhart (1983) studied theoretically and experimentally the stability and disturbance amplification produced by the combination of a natural convection flow induced by a vertical uniform heat flux surface and a forced convection flow given by a uniform free stream by assuming a weak forced convection flow with strong buoyancy effects. The authors presented stability planes and constant amplification contours, and found very good agreement between the two approaches. Daniels (1989) studied the stationary instability of the convective flow between differentially heated vertical planes and determined the subsequent structure of the neutral curve for stationary disturbances. Later, Chait and Korpela (1989) studied numerically the multicellular flow between two vertical parallel plates using a time-splitting pseudo spectral method for a steady flow of air and a timeperiodic flow of oil. In the case of air, the authors analyzed parametrically the three-dimensional linear stability of the flow and found that the domain of stable two-dimensional cellular motion is constrained by the Eckhaus instability and by two types of monotone instabilities. Their results show that the two-dimensional multicellular flow is unstable above a Grashof number of about 8550. Hence, the authors conclude that the flow of air in a sufficiently tall enclosure should be considered three-dimensional for most practical applications. Two decades ago, Rogers et al. (1993) studied the finite amplitude instability of mixed convection of air in a vertical concentric annulus with each cylinder maintained at a different temperature by use of 
weakly non-linear instability theory and direct numerical simulation. They found three different instabilities in the parameter space of Grashof and Reynolds numbers: one due to shear and the two others induced by thermal effects. Suslov and Paolucci (1995) studied the stability of mixed convection flow in a tall vertical channel under non-Boussinesq conditions and showed that the stability characteristics, such as the critical Grashof number and the disturbance wave speed, depend strongly on the temperature difference when fluid properties are allowed to vary. Chen and Chung $(1996,1998)$ studied the stability of a differentially heated vertical channel for various Prandtl numbers and showed that both the Prandtl and Reynolds numbers hold very important effects on the instability mechanism for high Prandtl number fluids. More recently, van Putten et al. (2001) presented experimental results of heat transfer processes in mixed convection from a ducted vertical hot-plate thermal flow sensor for aiding and opposing flows for three different values of the Grashof numbers, $G r=289,411$ and 456 for a range or Reynolds number from 0 to 120 . The authors found, in the transition from free to mixed and to essentially forced convection, distinct sequences of instabilities in the flow that lead to several local minima and maxima in the heat transfer from the plate. Cushman-Roisin (2005) proposed a non-usual approach to the Kelvin-Helmholtz instability by considering the latter as a boundaryvalue problem instead of using the traditional approach of an initial-value problem where wave perturbations of a two-layer shear flow grow over time into billows and eventually generate vertical mixing. His results show that although the wavelength and period of the critical wave differ from the classical problem, the outcome of the boundary-value problem is the same as for the initial-value configuration. In the last years, Guillet et al. (2007) considered the case of laminar mixed convection flow between vertical parallel plates heated uniformly by using a method based on the center manifold theorem to reduce the Navier-Stokes equations to ordinary differential equations in the vicinity of a trivial stationary solution and pointed out that when the forcing parameter or Rayleigh number increases beyond a critical value, the stationary solution is a pitchfork bifurcation point of the system. In the same year, Bera and Khalili (2007) used the linear theory of stability analysis to study numerically the impact of permeability on the stability of a buoyancy-opposed mixed convection in a vertical channel. They found that two main instability modes (Rayleigh-Taylor and buoyant instability) appear, and that for Darcy numbers $\leq 10^{-9}$, the Rayleigh-Taylor instability dominates within the entire Reynolds number range considered. In addition, they also found that for the same $R e$, the fully developed base flow is highly unstable (stable) for porous media with high (low) permeability, whilst the introduction of a one order of reduction in the permeability in the main flow direction made the system approximately 20 times more stable.

Although the oscillatory behavior of Navier-Stokes type systems in mixed convection has received relatively little attention, it is known that these flows can exhibit interesting dynamical phenomena. In many cases, the transition to turbulence is a chain of oscillatory states separated by bifurcations of different types. Examples where the bifurcation structure of such systems has been studied follow. Meron and Procaccia (1987) showed that in dynamical systems described by critical flows, the onset of chaos is via gluing bifurcations, and that these systems can be analyzed using discontinuous maps of the interval. The gluing bifurcation is a class of global bifurcations where, as an external control parameter is varied, two symmetrically related time-periodic states simultaneously become homoclinic to an unstable saddle state and result in a single symmetric time-periodic state Epstein and Pojman (1998); Ambruster et al. (1996); Abshagen et al. (2001). Arneodo et al. (1981) performed a study for one route to chaos via a cascade of bifurcations involving homoclinic orbits. Rucklidge (1993) described the transition to chaos through gluing processes in a three-dimensional magnetoconvection model. 
Marques et al. (2001) studied numerically a one-dimensional route in parameter space of a periodically forced flow with symmetry and provided a comprehensive analysis of the route to chaos, which involves a new and convoluted symmetry breaking that includes heteroclinic, homoclinic and gluing bifurcations. Lopez and Marques (2000) obtained three-tori solutions of the Navier-Stokes equations and their dynamics by use of a global Poincaré map. Their results show that these solutions undergo global bifurcations that include a new gluing bifurcation associated with homoclinic and heteroclinic connections to unstable solutions (two-tori) that act as organizing centers for the three-tori dynamics.

The foregoing survey of literature reveals that there are relatively few studies that address the thermal and flow stability in internal mixed convection for opposing flow in situations where flow reversal occurs. In the present study, a detailed numerical investigation is done of the symmetry breaking instability for laminar opposing mixed convection flow in a vertical channel of finite length subjected to isothermal and discrete heat inputs. For fixed Reynolds, Prandtl and geometry, the stability characteristics is to be obtained for increasing values of the buoyancy parameter.

\section{PROBLEM DESCRIPTION}

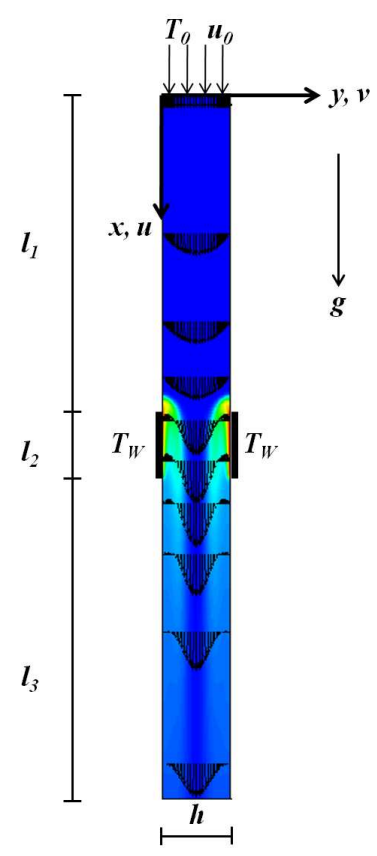

Figure 1: Schematic representation of the channel.

The loss of stability is studied for a symmetrical counter-current mixed convection problem inside a vertical channel of finite length with a flat velocity distribution at the channel entrance, with the channel walls heated discretely and symmetrically. The schematic view of the geometry considered is shown in Fig. 1. The forced flow is driven by gravitational force acting vertically downward, entering the duct with a uniform velocity $u_{0}$ and ambient temperature $T_{0}$. Axial distances from the entrance section are measured by the $x$ coordinate (positive downward), while transverse distances are measured by $y(y=0$ at the left wall). Both walls, separated by a distance $h$ have discrete heat sources of length $l_{2}$ located at $x=l_{1}$, with uniform wall temperature $T_{w}$, where $T_{w}>T_{0}$. All other surfaces of the channel walls are assumed adiabatic 
insulators. Flow rectifiers are placed at the channel entrance and exit, thus producing a parallel flow at $x=0$ and $x=l_{1}+l_{2}+l_{3}$. The viscous dissipation in the energy equation is neglected and the thermo-physical properties of the fluid are assumed to be constant except for the density in the buoyancy term, which is treated according to the Boussinesq approximation. By the use of the vorticity $(\Omega=\partial V / \partial X-\partial U / \partial Y)$ and stream function formulation $(U=\partial \psi / \partial Y$, $V=-\partial \psi / \partial X)$, the flow is described by the non-dimensional equations

$$
\begin{gathered}
\frac{\partial^{2} \psi}{\partial X^{2}}+\frac{\partial^{2} \psi}{\partial Y^{2}}=-\Omega \\
\frac{\partial \Omega}{\partial \tau}+\frac{\partial \psi}{\partial Y} \frac{\partial \Omega}{\partial X}-\frac{\partial \psi}{\partial X} \frac{\partial \Omega}{\partial Y}=\frac{1}{\operatorname{Re}}\left(\frac{\partial^{2} \Omega}{\partial X^{2}}+\frac{\partial^{2} \Omega}{\partial Y^{2}}\right)-R i \frac{\partial \theta}{\partial Y} \\
\frac{\partial \theta}{\partial \tau}+\frac{\partial \psi}{\partial Y} \frac{\partial \theta}{\partial X}-\frac{\partial \psi}{\partial X} \frac{\partial \theta}{\partial Y}=\frac{1}{\operatorname{RePr}}\left(\frac{\partial^{2} \theta}{\partial X^{2}}+\frac{\partial^{2} \theta}{\partial Y^{2}}\right)
\end{gathered}
$$

where $\vec{V}=(U, V)$ is the dimensionless velocity vector and $\theta$ is the dimensionless temperature. In the above equations, all velocity components ( $U$ in the $X$-direction and $V$ in the $Y$-direction) are scaled with the inflow velocity, $u_{0}, U=u / u_{0}$ and $V=v / u_{0}$; the longitudinal coordinates are scaled with the channel width $h, X=x / h$ and $Y=y / h$; the time is scaled with the residence time $h / u_{0}, \tau=t u_{0} / h$; the temperature is normalized as $\theta=\left(T-T_{0}\right) /\left(T_{w}-T_{0}\right)$. The nondimensional parameters appearing in the above equations are the Reynolds number, $R e=u_{0} h / v$ (inertial to viscous forces), the Prandtl number, $\operatorname{Pr}=v / \alpha$ (viscous to thermal diffusivities) and the Richardson number, $R i=g \beta\left(T_{w}-T_{0}\right) h / u_{0}^{2}$ (buoyancy induced potential to forced kinetic energy). Here, $g$ is the gravity acceleration and $\beta$ is the thermal expansion coefficient. Another buoyancy parameter employed frequently is the Grashof number, $G r=R i R e^{2}$, which relates buoyancy to viscous forces. Additional non-dimensional geometrical parameters arise through the boundary conditions, $L_{i}=l_{i} / h$, with $i=1,3$. Eqs. (1) to (3) have to be solved with the following boundary conditions:

Uniform flow at the channel entrance:

$$
\psi(0, Y)-Y=\Omega(0, Y)=0,
$$

no slip at the walls,

$$
\begin{gathered}
\psi(X, 0)=\Omega(X, 0)-2(\psi(X, 0)-\psi(X, \Delta Y)) / \Delta Y^{2}=0, \\
\psi(X, 1)-1=\Omega(X, 1)-2(\psi(X, 1)-\psi(X, 1-\Delta Y)) / \Delta Y^{2}=0,
\end{gathered}
$$

and relaxed parallel flow conditions at the chancel exit:

$$
\partial \psi /\left.\partial X\right|_{X=L}=\partial^{2} \psi /\left.\partial X \partial Y\right|_{X=L}=0,
$$

where $L$ is the total length of the channel, $L=L_{1}+L_{2}+L_{3}$. The boundary conditions for temperature are the following:

Fixed temperature at the heated sections

$$
\theta=1 \text { at } Y=Y_{w} \text { with } L_{1} \leq X \leq L_{1}+L_{2} .
$$


Adiabatic channel walls are considered,

$$
\partial \theta / \partial Y=0 \text { at } Y=Y_{w} \text { except in } L_{1} \leq X \leq L_{1}+L_{2} .
$$

The dynamical properties of the system are described using the average non-dimensional heat fluxes or Nusselt numbers at both heated plates, $\mathrm{Nu}_{L, R}=\left|\bar{q}_{L, R}\right| h /\left(k\left(T_{w}-T_{0}\right)\right)$ and the nondimensional first moment of the longitudinal velocity, $Y_{p}=\left(1 / h^{2} u_{0}\right) \int_{0}^{h} y u d y$. Here $k$ is the thermal conductivity of the fluid. $\mathrm{Nu}_{L, R}$ depend only on time and $Y_{p}$ is a function of the longitudinal position and time. Due to baroclinity -last term of Eq. (2)-, vorticity is produced and vortices (large recirculation bubbles) are generated. The position of the recirculation zones are represented by a stagnation point at $X=X_{S}(\tau)$, defined by the maximum value of $X$, where the longitudinal velocity component is non-negative in the vortex region. Eqs. (1) to (3) are numerically solved using a strongly non-uniform staggered grid system with a denser clustering near the heated plate. The technique employed has been described elsewhere Martínez-Suástegui et al. (2011). In this work the Reynolds number is $R e=100$, with $l=12 h$. The length of the heated slabs is $l_{2}=h$, and they are located at $x=l_{1}=5.5 h$, that is $l_{1}=l_{3}=5.5 h$. The non-dimensional time step $\Delta \tau=u_{0} \Delta t / h$ has been set to $5 \times 10^{-4}$, and computation is terminated when the time evolution of the system state reaches steady-state or a final self-sustained oscillatory state.

\section{NUMERICAL SOLUTION}
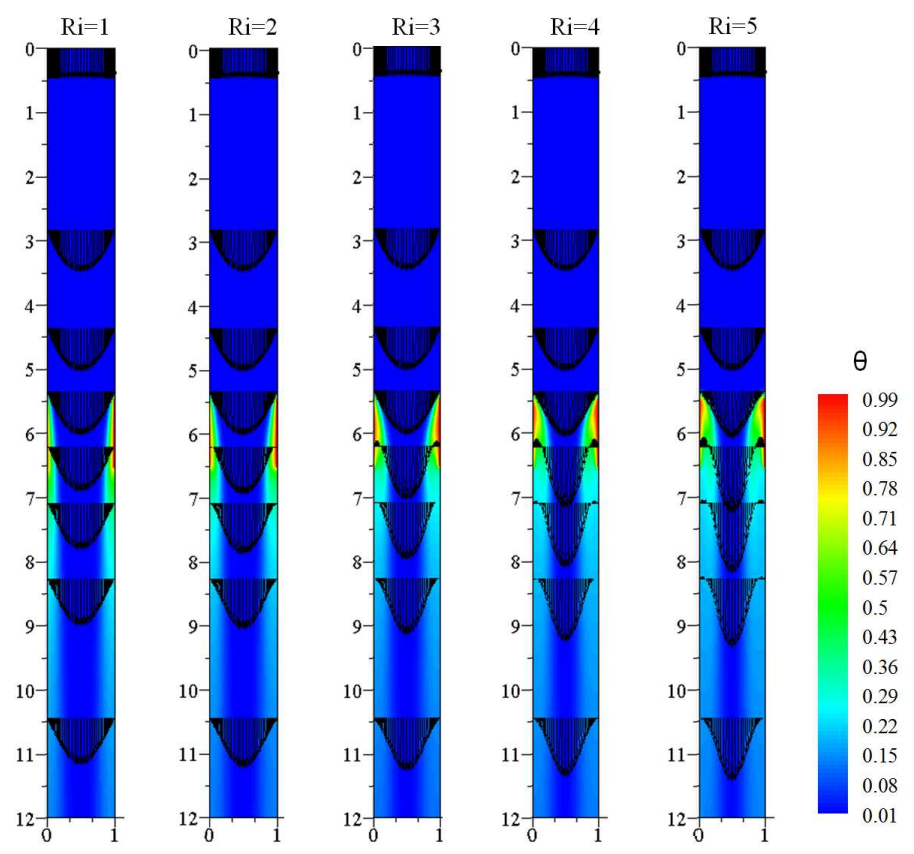

Figure 2: Final states for a steady and symmetrical dynamical response of the system.

After switching buoyancy on and for relatively small values of the Richardson number, the flow reverses close to the heated slabs and a pair of symmetric vortices develop due to baroclinity. Both vortices reach a maximum position represented by the stagnation point, which decreases (higher positions) as the buoyancy parameter increases. There is an equilibrium between buoyancy in the hot recirculation bubble and the dynamical pressure and drag from the 
cold downward fluid. For a range of values of the Richardson number of $R i<5.2$, the transient response leads to a symmetrical steady state solution. For these relatively low Richardson numbers there is only a weak interaction in the dynamics of both recirculation bubbles. Figure 2 shows the velocity and temperature profiles after the dynamical system has reached a symmetrical steady state. For this range of the buoyancy parameter, the final states of the system correspond to steady symmetric flow. As the Richardson number increases, small amplitude flow oscillations appear in the downstream region of the channel and their amplitude increase for increasing values of the buoyancy parameter. These flow oscillations are a manifestation of the Kelvin-Helmholtz instability due to the strong shear between the ascending hot fluid and the descending cold fluid located in the middle of the channel. This triggers a symmetrybreaking bifurcation and one of the two vortices (assumed to be close to the left heated surface, for simplicity) climbs while the other is pushed down by the downward flow with increased longitudinal momentum. If one vortex climbs, the cold fluid deflects to the other side of the channel increasing the longitudinal velocity and thus the momentum, pushing down the other vortex. As the fluid reaches the top of the lower vortex, the cold downward fluid switches sides again, thus supporting the upper recirculation bubble. Crude numerical simulations show that the symmetry breaking bifurcation occurs for a Richardson number of $R i=5.2$. As a result, a stable non-symmetric pattern develops after a relatively short transient. Fig. 3 shows the resulting evolution of the overall Nusselt number for the specific case of a Reynolds number of 100 and a Prandtl number of 7. As the Richardson number increases, the Nusselt number first decreases and later increases. This behavior is due to the change of the flow direction close to the wall. For a Richardson number close to 5.2 symmetry breaks and one of the recirculation bubble climbs while the other is pushed down, producing two solution branches for both hot surfaces. If now, the Richardson number decreases, the response continues to be asymmetric for values down to 4.1, where suddenly the symmetric behavior is recovered. This hysteresis loop is a clear indication of a subcritical bifurcation process to be analyzed below.

\section{LINEAR STABILITY ANALYSIS}

For a given fixed Reynolds number and small values of the Richardson number, a symmetrical flow response is obtained. In order to study the stability of the symmetrical flow, a symmetrical strongly non-parallel base flow with variables denoted by $\psi_{0}, \Omega_{0}$ and $\theta_{0}$ as functions of the Richardson number, is numerically generated, given by Eqs. (1) to (3), but with the following symmetrical boundary conditions:

Uniform flow at the channel entrance:

$$
\psi_{0}(0, Y)-Y=\Omega_{0}(0, Y)=\theta_{0}(0, Y)=0,
$$

no slip at the wall,

$$
\psi_{0}(X, 0)=\Omega_{0}(X, 0)-2\left(\psi_{0}(X, 0)-\psi_{0}(X, \Delta Y)\right) / \Delta Y^{2}=0,
$$

symmetry conditions at the symmetry plane $(Y=0.5)$,

$$
\psi_{0}(X, 0.5)-0.5=\Omega_{0}(X, 0.5)=\partial \theta_{0} / \partial Y=0
$$

and relaxed parallel flow conditions at the channel exit,

$$
\partial \psi_{0} /\left.\partial X\right|_{X=L}=\partial^{2} \psi_{0} /\left.\partial X \partial Y\right|_{X=L}=0
$$




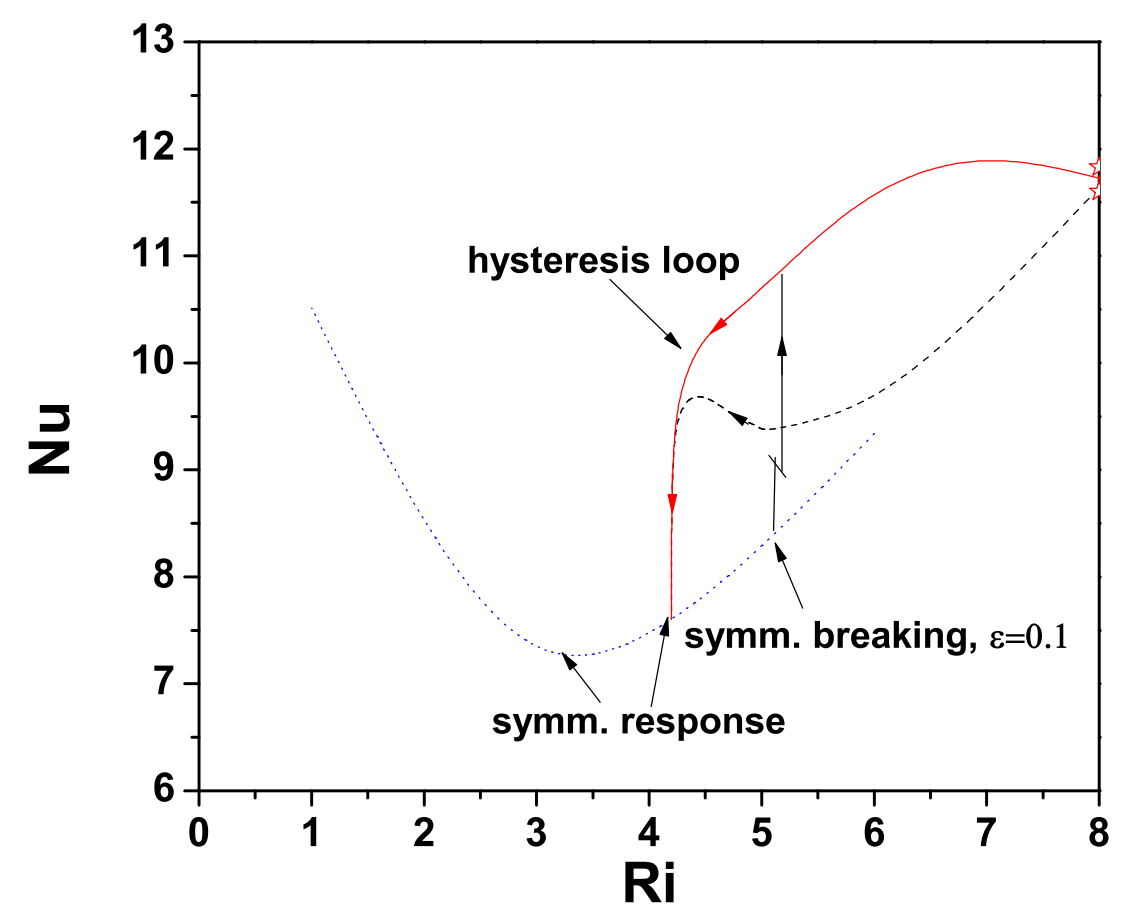

Figure 3: Hysteresis behavior for $\operatorname{Re}=100$ and $\operatorname{Pr}=7$.

A solution of the form $\Omega=\sum_{i=0}^{\infty} \delta^{n} \Omega_{n}, \psi=\sum_{i=0}^{\infty} \delta^{n} \psi_{n}$ and $\theta=\sum_{i=0}^{\infty} \delta^{n} \theta_{n}$, is assumed, with $\delta \rightarrow 0$. The linearized first order equations are

$$
\begin{aligned}
& \frac{\partial \Omega_{1}}{\partial \tau}+\frac{\partial \psi_{0}}{\partial Y} \frac{\partial \Omega_{1}}{\partial X}-\frac{\partial \psi_{0}}{\partial X} \frac{\partial \Omega_{1}}{\partial Y}-\frac{1}{R e} \nabla^{2} \Omega_{1}= \\
&-\frac{\partial \psi_{1}}{\partial Y} \frac{\partial \Omega_{0}}{\partial X}+\frac{\partial \psi_{1}}{\partial X} \frac{\partial \Omega_{0}}{\partial Y}-R i \frac{\partial \theta_{1}}{\partial Y} \\
& \nabla^{2} \psi_{1}=-\Omega_{1}, \\
& \frac{\partial \theta_{1}}{\partial \tau}+\frac{\partial \psi_{0}}{\partial Y} \frac{\partial \theta_{1}}{\partial X}-\frac{\partial \psi_{0}}{\partial X} \frac{\partial \theta_{1}}{\partial Y}-\frac{1}{\operatorname{RePr}} \nabla^{2} \theta_{1}=-\frac{\partial \psi_{1}}{\partial Y} \frac{\partial \theta_{0}}{\partial X}+\frac{\partial \psi_{1}}{\partial X} \frac{\partial \theta_{0}}{\partial Y},
\end{aligned}
$$

with the required homogeneous boundary conditions in the whole channel. A non trivial solution can be obtained from numerically generated eigenfunctions, using the perturbed Richardson number

$$
R i_{\varepsilon}=R i\left(1+\varepsilon e^{-\tau / \tau_{0}} f(y)\right),
$$

with two different modes: $f(y)=\sin (2 \pi y)$ or $f(y)=\cos (\pi y)$. In the above relation $\tau_{o}$ is set of order unity and $\varepsilon$ is a parameter with a value very small compared with unity. The non-symmetric forcing relaxes the solution in a short time to a numerical eigenfunction, which evolves with time, giving the stability conditions by detecting the amplitude evolution of the overall Nusselt perturbation and its oscillation frequency, $N u_{1}=\int_{L_{1}}^{L_{1+1}} \partial \theta_{1} /\left.\partial Y\right|_{Y=0} d X \sim \exp (\sigma+$ $i S) \tau$, where $i=(-1)^{1 / 2}$. Positive values of $\sigma$ means instability. $S$ is then the non-dimensional 


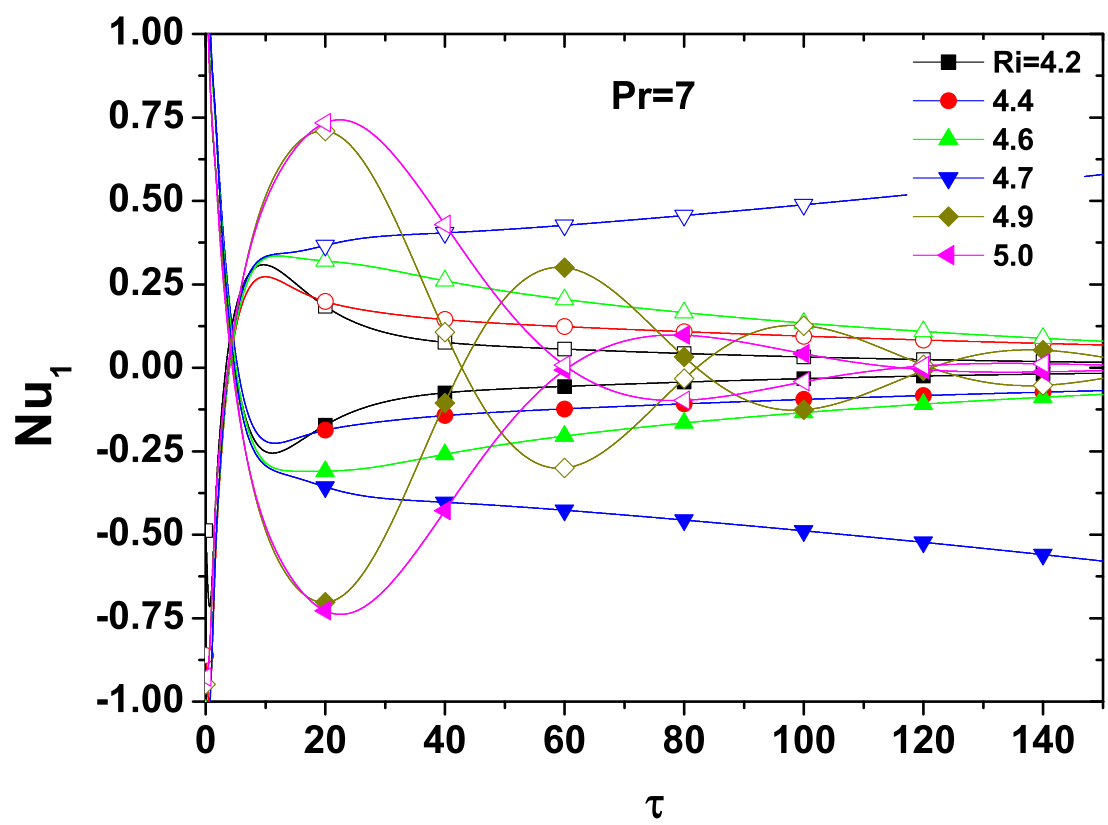

Figure 4: Time evolution of the perturbed Nusselt number for $\operatorname{Pr}=7$ and different values of the Richardson numbers from 4.2 to 5.0 .

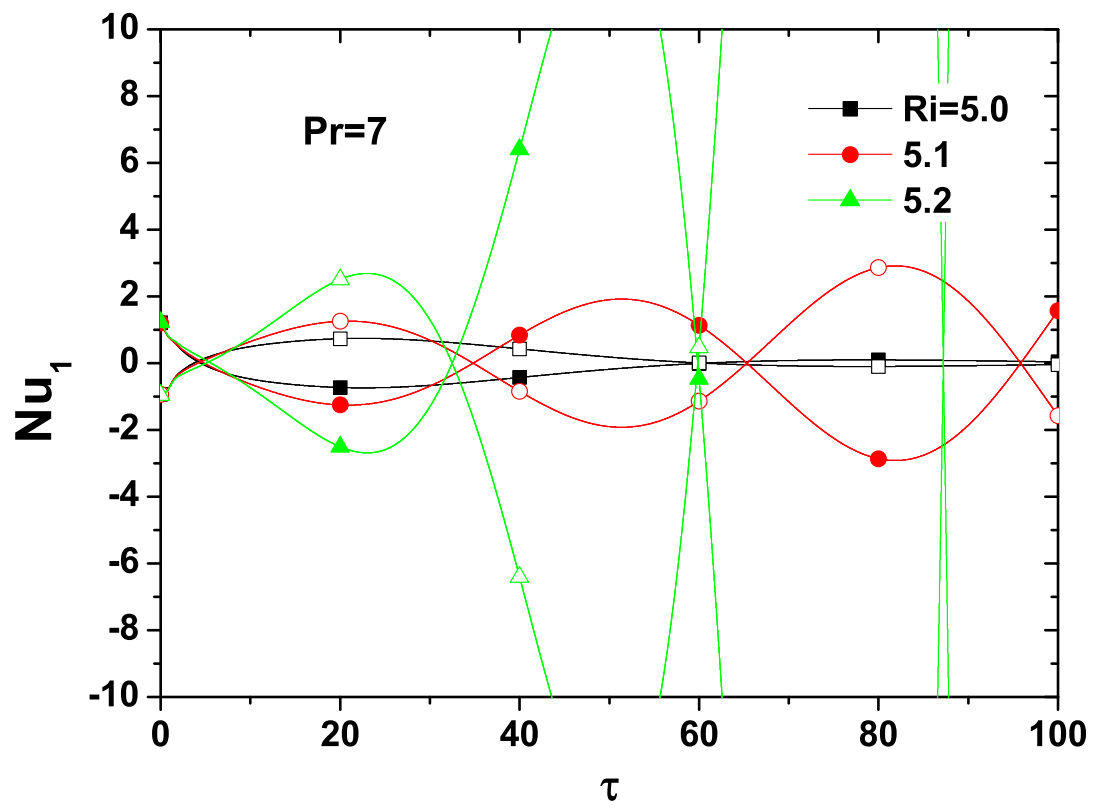

Figure 5: Time evolution of the perturbed Nusselt number for $\operatorname{Pr}=7$ and different values of the Richardson numbers from 5.0 to 5.2 .

oscillation frequency (Strouhal number). Fig. 4 shows the time evolution of the perturbed Nusselt number $N u_{1}$ for a Prandtl number of $\operatorname{Pr}=7$ at both heated surfaces, for different values of the Richardson numbers up to $R i=5.0$. For Richardson numbers up to 4.6 the perturbed solu- 


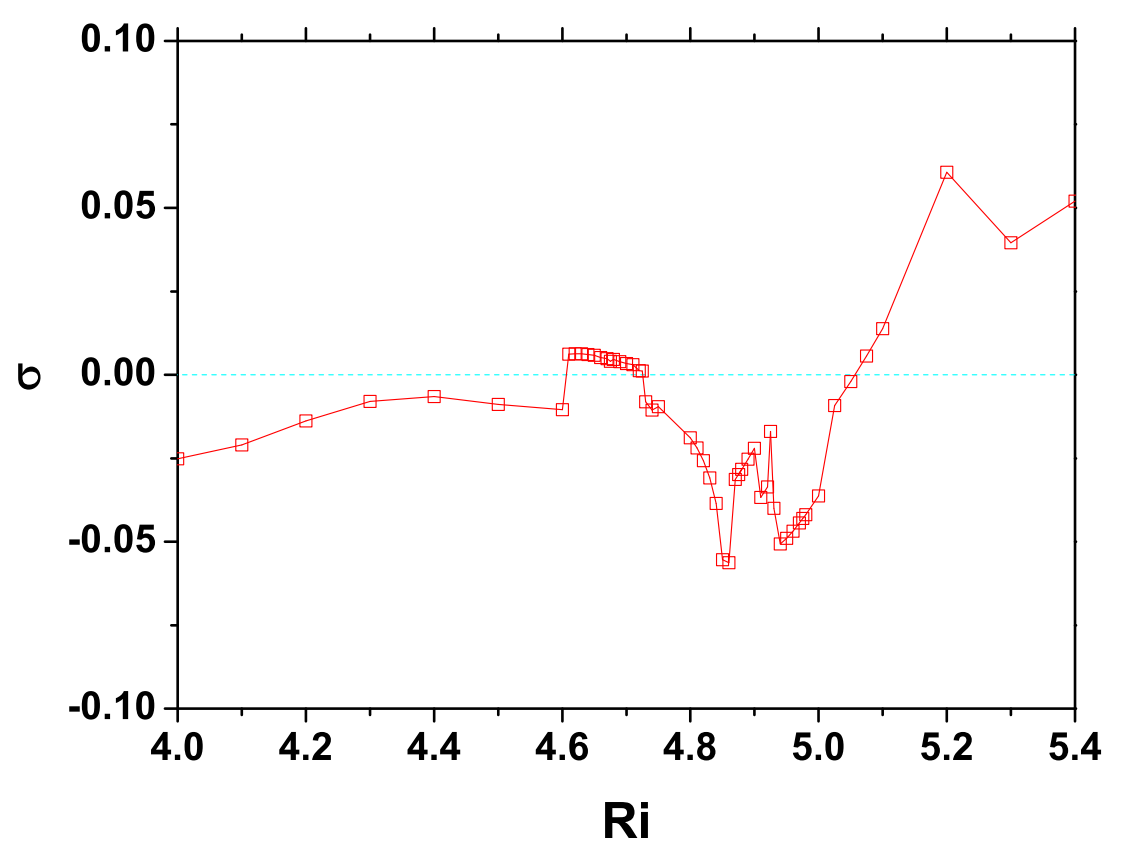

Figure 6: Growth rate as a function of the Richardson number for $\operatorname{Pr}=7$. Positive values means instability.

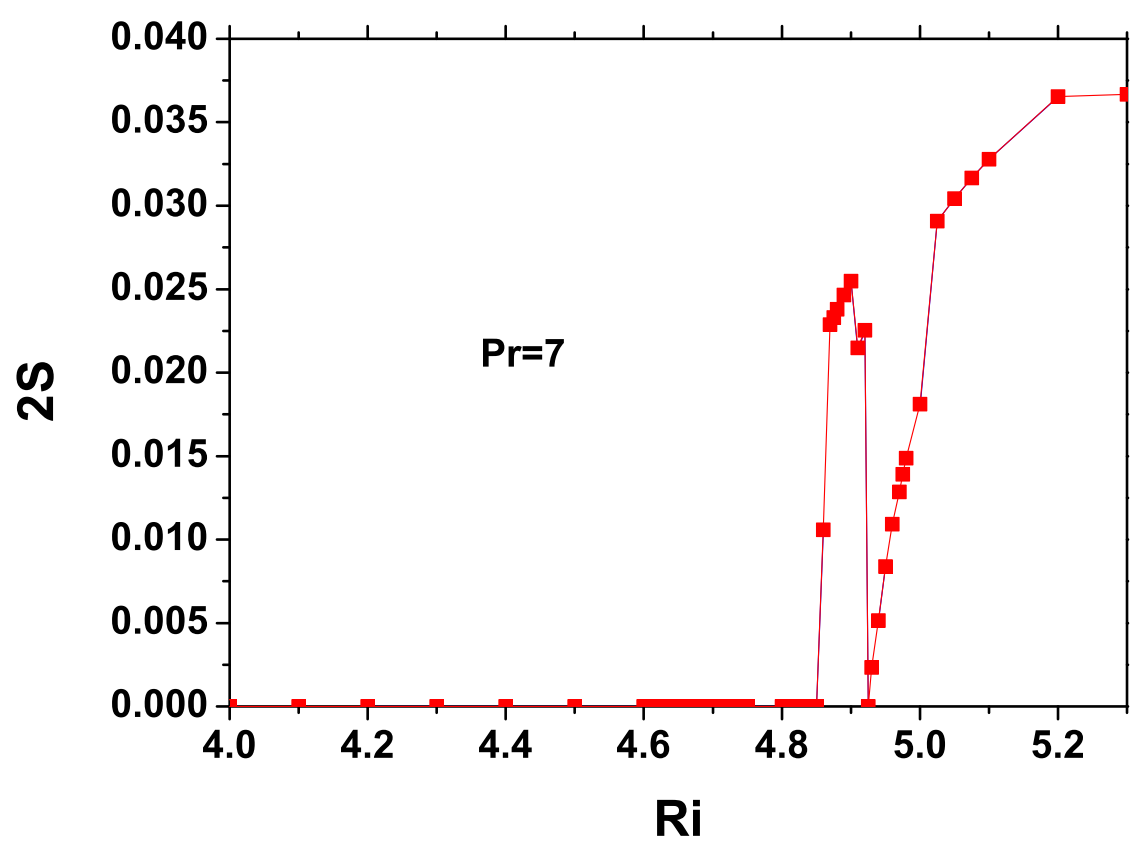

Figure 7: Oscillation frequency of the linear perturbation as a function of the Richardson number for $\operatorname{Pr}=7$.

tion decays without oscillating. On the other hand for values between 4.9 and 5.0 the response solution decays oscillating with different frequencies and decay rates. However, surprisingly, for a Richardson number of 4.7, the amplitude perturbed solution increases monotonically with time, which is indication that for this particular case, the symmetrical flow is unstable. Using the 
non-linear equations it can be shown that a slightly non-symmetric steady state flow response is obtained for this Richardson number. For Richardson numbers above 5.0, Fig. 5 shows the time evolution of the perturbed Nusselt number. Here again, for $R i=5.0$ the stable decaying oscillating response is depicted. As the Richardson number increases, the oscillating frequency also increases. For Richardson numbers $R i \geqq 5.1$ the system is now unstable. Figs. 6 and 7 show the the real $(\sigma)$ and the imaginary $(S)$ parts, respectively, of the exponential growth, for a Prandtl number of $P r=7$. The system is linearly unstable in the ranges $4.606<R i<4.72$ and $R i>5.06$. Instability leads in both cases to steady nonsymmetric thermal and flow configurations with two vortex structures at different heights. Although the symmetrical flow configuration is unstable for values of $4.606<R i<4.72$, soon non-linearities limit the growth of perturbations producing a slight modified asymmetrical steady thermal and flow pattern, where the recirculation bubbles only move apart slightly in the vertical position. However, for values of $R i>5.06$, the difference of the maximum height of both vortical structures at the final steady state is of the order of the channel width, that is of order unity in non-dimensional units. Fig. 7 shows that the time evolution of the perturbation is monotonic for $R i<4.85$ and oscillatory for larger values of the Richardson number, except for a very thin region around $R i=4.92$, where the oscillation frequency reduces drastically. This atypical response arises because for each Richardson number, a different base flow is needed.

\section{CONCLUSION}

In this work, the linear stability of a two dimensional symmetrical counter-current mixed convection system is analyzed using numerically generated eigenfunctions. The problem depends on several non-dimensional parameters like the flow Reynolds number, the Prandtl number of the fluid, the Richardson number (buoyancy parameter) and geometrical parameters of the vertical channel. The symmetry breaking instability has been obtained for fixed geometry, Reynolds $(R e=100)$ and Prandtl $(P r=7)$ numbers, by increasing the buoyancy parameter. For low Richardson numbers $(R i<4.606)$, the resulting flow is steady and symmetric with two vortical flow structures anchored slightly above the heated sources. The system shows an instability for the range $4.606<R i<4.72$, where the two vortical structures separate only slightly in the vertical position. The steady-state symmetrical response is again obtained for larger Richardson numbers up to $R i=5.06$, where again the system looses stability, producing a much larger separation of the vortices in the vertical coordinate.

\section{REFERENCES}

Abshagen J., Pfister G., and Mullin T. Gluing bifurcations in a dynamically complicated extended flow. Phys. Rev. Lett., 87:224501, 2001.

Ambruster D., Nicolaenko B., Smaoui N., and Chossat P. Symmetries and dynamics for 2d navier-stokes flow. Physica D, 95:81-93, 1996.

Arneodo A., Coullet P., and Tresser C. A possible new mechanism for the onset of turbulence. Phys. Lett., 81A:197, 1981.

Bera P. and Khalili A. Stability of buoyancy opposed mixed convection in a vertical channel and its dependence on permeabilty. Adv. Water Res., 30:2296-2308, 2007.

Carey V.P. and Gebhart B. The stability and disturbance-amplification characteristics of vertical mixed convection flow. Journal of Fluid Mechanics, 127:185-201, 1983.

Chait A. and Korpela S.A. The secondary flow and its stability for natural convection in a tall vertical enclosure. Journal of Fluid Mechanics, 200:189-216, 1989. 
Chang T.S. and Lin T.F. Steady and oscillatory opposing mixed convection in a symmetrically heated vertical channel with a low-prandtl number fluid. Int. J. Heat Mass Transfer, 36:37833795, 1993.

Chen Y.C. and Chung J. The linear stability of mixed convection in a vertical channel flow. $J$. Fluid Mech., 325:29-51, 1996.

Chen Y.C. and Chung J. Stability of mixed convection in a differentially heated vertical channel. ASME J. Heat Transfer, 120:127-132, 1998.

Cushman-Roisin B. Kelvin-helmholtz instability as a boundary-value problem. Environmental Fluid Mechanics, 5:507-525, 2005.

Daniels P. Stationary instability of the convective flow beetween differentially heated vertical planes. J. Fluid Mech., 203:525-540, 1989.

Epstein I. and Pojman J. An introduction to nonlinear chemical dynamics: Oscillations, waves, patterns, and chaos. Oxford University Press, 1998.

Evans G. and Greif R. Buoyant instabilities in downward flow in a symmetrically heated vertical channel. Int. J. Heat Mass Transfer, 40:2419-2425, 1997.

Guillet C., Mare T., and Nguyen C.T. Application of a non-linear local analysis method for the problem of mixed convection instability. International Journal of Non-Linear Mechanics, 42:981-988, 2007.

Lin T.F., Chang T.S., and Chen Y.F. Development of oscillatory asymmetric recirculating flow in transient laminar opposing mixed convection in a symmetrically heated vertical channel. ASME J. Heat Transfer, 115:342-352, 1993.

Lopez J. and Marques F. Dynamics of three-tori in a periodically forced navier- stokes flow. Phys. Rev. Lett., 85:972-975, 2000.

Lorenz E.N. Deterministic non-periodic flow. Journal of Atmospheric Sciences, 20:130-141, 1963.

Marques F., Lopez J., and Shen J. A periodically forced flow displaying symmetry breaking via a three-tori gluing bifurcation and two-tori resonances. Physica D, 156:81-97, 2001.

Martínez-Suástegui L. and Treviño C. Particle image velocimetry measurements for opposing flow in a vertical channel with a differential and asymmetric heating condition. Exp. Therm. Fluid Sci., 32:262-275, 2007.

Martínez-Suástegui L., Treviño C., and Cajas J. Thermal nonlinear oscillator in mixed convection. Phys. Rev. E, 84:046310, 2011.

Meron E. and Procaccia I. Gluing bifurcations in critical flows: The route to chaos in parametrically excited surface waves. Phys. Rev. A, 35:4008-4011, 1987.

Rogers B.B., Moulic S.G., and YAO L.S. Finite-amplitude instability of mixed convection. Journal of Fluid Mechanics, 254:229-250, 1993.

Rucklidge A. Chaos in a low-order model of magnetoconvection. Physica D, 62:323-337, 1993.

Suslov S. and Paolucci S. Stability of mixed-convection flow in a tall vertical channel under non-boussinesq conditions. J. Fluid Mech., 302:91-115, 1995.

van Putten M.J.A.M., Kleijn C.R., and van den Akker H.E.A. Heat transfer and temporal behavior of the laminar mixed-convection flow around a ducted flat-plate thermal flow sensor. Experimental Heat Transfer, 14:229-250, 2001. 